\title{
Interaction Should Guide Management Decisions
}

l: $\mathrm{t}$ is with great interest that we read the recent article by Jadhav et $\mathrm{al}^{1}$ concerning subgroup assessments in the SWIFT PRIME trial, especially for collateral status. They reported, "Outcomes stratified by collateral status had a benefit with thrombectomy across all groups: none-to-fair collaterals ( $33 \%$ versus $0 \%$ ), good collaterals ( $58 \%$ versus $44 \%$ ), and excellent collaterals ( $82 \%$ versus $28 \%$ )." They also reported, "A beneficial effect of endovascular therapy was observed over IV tPA alone across all levels of collateral flow, with the greatest effect in patients with excellent collaterals (82\% versus $28 \%, P=$ .008)."

The authors reported that benefit persisted across multiple subgroups, but they still concluded, "Overall, this report supports the selection of patients for intra-arterial therapy on the basis of favorable patient characteristics (small core, good collateral circulation)." We do not agree with this conclusion because these patient characteristics were, foremost, assessed for prognosis, not treatment effect. Tests for statistical interaction between the predictors (treatment allocation arm and subgroups defined post hoc) were not performed, making the interpretation of data difficult.

The value and role of interaction testing and why it is not appropriate to test for superiority of a treatment in single subgroups are well presented in a Lancet educational series. ${ }^{2}$ In short, interaction evaluates whether the combination of 2 predictors affects the outcome in other ways than expected by each variable alone. To test for superiority in single subgroups is inappropriate because both falsenegative (sample size reduced) and false-positive (multiple testing) findings are possible.

In the current example, having treatment was better than not having treatment, ${ }^{3}$ and having good collaterals was better than having bad collaterals - both among those treated and among those not treated. ${ }^{1}$ Thus, it seems that treatment is effective regardless of collateral status, and no obvious interaction is observed. A reasonable alternative hypothesis to test would have been that those with poor collaterals would do poorly, both with and without treatment; thus, the subgroup affects the treatment effect, which would be the basis for an interaction. For the sake of argument, let us assume that these results instead came from a large sample, that formal interaction tests had been performed, and that no interaction was detected. In such circumstances, patient characteristics would have affected prognosis, but not treatment effect, an important distinction: To treat those with good collaterals will result in excellent outcome in the eyes of the interventionist (high share of recoveries), but to treat regardless of collateral status will create more patient benefit (because all benefit) but a worse outcome in the eyes of the interventionist (lower share of recoveries). We advocate that the latter is more relevant, but this is not consistent with the authors' conclusions.

However, given the current small sample of 109 patients, divided into 6 groups, the risk of false-negative findings for interaction is high. The finding that having good collaterals was associated with a better outcome in the IV tPA arm compared with having excellent collaterals in the IV tPA arm further supports the study being underpowered and findings possibly being spurious and/or the result of multiple testing.

We encourage the authors to merge their data on a patient level with those of other recent similar trials so that these analyses will be well-powered and meaningful. We advocate against clinical decision-making based on the current preliminary data.

Disclosures: Elias Johansson—UNRELATED: Grants/Grants Pending: Several grants totaling $\$ 520,000$, all from nonprofit research bodies, the majority from the Swedish Government: Västerbotten County $(\$ 400,000)$, Neurological Research Fund at Umeå University Hospital, Northern Swedish Stroke Fund, National Swedish Stroke fund, Jeansson Foundation*; Royalties: text books, Comments: about $\$ 100-\$ 200$ annually in royalties for 2 book chapters in a stroke book in Swedish. Jonatan Salzer-UNRELATED: Grants/Grants Pending: Synapsys, Comments: nystagmus investigation equipment donated for research*; Stock/Stock Options: Eurocine Vaccines, SensoDetect, Comments: stocks worth $\$ 130$ in 2 biomed starter companies making flu vaccines (Eurocrine Vaccines) and neuropsychiatric diagnostic equipment (Sensodetect) ${ }^{\star}$ *Money paid to the institution.

\section{REFERENCES}

1. Jadhav AP, Diener HC, Bonafe A, et al; SWIFT PRIME Investigators. Correlation between clinical outcomes and baseline CT and CT angiographic findings in the SWIFT PRIME trial. AJNR Am J Neuroradiol 2017;38:2270-76 CrossRef Medline

2. Rothwell PM. Treating individuals 2: subgroup analysis in randomised controlled trials - importance, indications, and interpretation. Lancet 2005;365:176-86 CrossRef Medline

3. Saver JL, Goyal M, Bonafe A, et al; SWIFT PRIME Investigators. Stentretriever thrombectomy after intravenous t-PA vs. $t-P A$ alone in stroke. N Engl J Med 2015;372:2285-95 CrossRef Medline

(D. Johansson (D). Salzer Pharmacology and Clinical Neuroscience Umeå University Umeå, Sweden 